\title{
Innovation of methodic "colour associations of letter-signs ", worked out according to self organization system concept
}

\author{
Ludmila V. Akhmetova \\ Tomsk State Pedagogical University \\ Tomsk, Russia \\ axmetova@tspu.edu.ru
}

\begin{abstract}
The article is devoted to the description of the authentic methodic "Colour Associations of Letter-Signs" (CALS), held on-line, its methodological basis is the concept of person cognitive sphere (PCS) self organization. PCS is which can be described as unique united plurality constructed on the basis of triunique principle. The main procedure is measuring of the sensor-perception activity of a person, which can be found as difference between free (realized) and projective (unrealized) color associations of the Russian letter sings, through the analysis of the frequency marks of the letter-sign color specter of the individual (original) text.
\end{abstract}

Keywords - methods, colour associations, letter signs, cognitive sphere of personality, system.

\section{INTRODUCTION}

The self organization concept is a perspective approach in understanding the way of how cognitive systems are functioning. Synergy as a fundamental property of self organizational systems gives a possibility to explain appearance of these systems new properties on the basis of systems elements correlation. According to this scientific point of view we can consider the phenomenon of spontaneous forming of order in cognitive structures. The order is represented in human consciousness and is used to perceive the world in the form of subjective images. The reader will probably observe that self organizational systems concept as it is has long history in this field of science and there are already a lot of discoveries, science and applied works. But at the same time questions concerning intellectual activity and especially cognitive one have not full and direct answers. In the present article the author tries to cover one of the aspects of human cognitive activity from the point of view of the self organizational systems theory, and the author suggests a short scheme describing the concept of person cognitive sphere structural organization, and one of the methods of diagnostic investigations called "Method of Color Associations of Letter-Signs" (MCALS).

\section{MAIN PART}

The data obtained in the course of investigation of schoolchildren allow us to define two determinants (or substructures) which form main directions in intellectual development [1-3]. The first one is "perception and mnemonic" determinant (PMD) and the second one is "mental" determinant (MD). It was also discovered that the determinants structure is dynamic and is possible for further complication. On the one hand this complication originates from new inner perception and mnemonic and mental connections, and on the other hand it happens due to the growth of connections between perception and mnemonic complex elements and definite mental complex indications. In the course of investigation it was also proved that the process of perception and mnemonic complex connections development has stronger intensity towards forming correlations with logical thinking, comparison, analysis and knowledge, which are included into the MD structure. PMD represents structural peculiarities of person natural development and includes 5 indications investigating. They are attention, visual memory, auditory memory, visual perception, auditory perception, correlates in the greatest degree with non-verbal individual psychological person peculiarities. MD according to its matter (space thinking, creative thinking, associative thinking, logical thinking, planning in mind, combination abilities, knowledge of the environment, mental operations such as comparison, analysis, generalization, abstract) corresponds to verbal aspect.

In the Fig. 1 there is a person cognitive sphere (PCS) general model. This model functioning effectiveness is provided by organization specifics of this model structure. The present model includes minimal and enough set o elements which are necessary for system growth and development. The first element is evolutional self organizational potential (ESOP), human nature, non-verbal aspect. According to cognitive indications structural organization it is "perception and mnemonic" determinant $[1,2]$. The second element is semiotic system (SS), verbal aspect, mind, "mental" determinant $[1,2]$. The third element is activity. Each separate element presents definite system. Structural elements are other components or elements of the second order, etc. Coordinated functioning of elements multitude of the united PCS system defines its dynamics. When investigating PMD PCS structure it was determined that structural components are able to build typical ordered and at the same time easily reconstructed cognitive formations. PMD parameters change under some cognitive influences and it is followed by structural reconstruction of "perception and mnemonic" complex (PMC). It changes its functioning order and leads to new quantitative and qualitative level. In such conditions we may say that the basic components of PCS are PMD and MD, which are 
characterized by dynamic. It means they can change under the influence of inner and outer factors having different origin. And PCS in the whole and separately represent open dynamic system self developing and self renovated [1].During our investigation we also proved that thinking productivity is reasoned not only by cognitive abilities balance but in the greatest degree by PCS structural organization and its basic components that are cognitive development determinants. The optimal balance of quantitative and qualitative results of structural organization is able to develop constant positive path of cognitive development and individual thinking productivity [3].

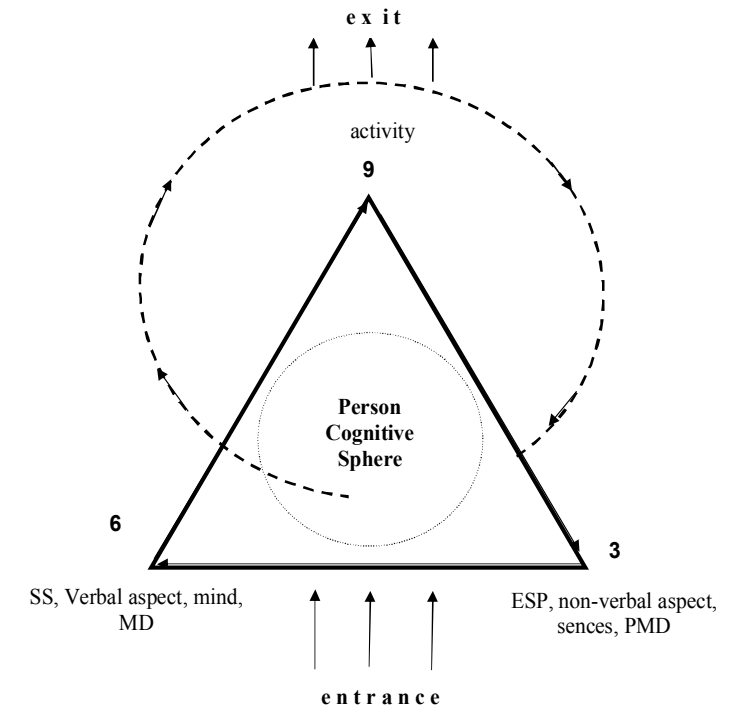

Fig 1. General view of the PCS model.

We created computer version of this methodic according to the idea of PCS as self organizational system and on the basis of discovered individual thinking productivity dependence on the optimal balance of quantitative and qualitative PCS structural organization results.

MCALS novelty and originality. The probationers associate preferable colors with definite letters of their individual frequency letter-sign specter. We obtain and apply results to the psycho-diagnostic investigation. The individual frequency letter-sign specter reveals in the authentic text, which the probationers write during the test. Thus data obtained on the ground of natural (non-verbal) and formal (verbal) integration undergo comparative analysis and estimation. The aim of MCALS is to reveal the number of person individual psychological peculiarities using the method of psychological investigation. The test procedure includes value measuring of person sensorperception adequacy, which can be found as disparity between deliberate color associations of Russian alphabet letters displayed on realized level (color preferences in the form of scheme building), color attribution (social and cultural attitudes) and color associations on the projective (unrealized) level. It is reached by means of frequency markers analysis of the original text letter-signs specter color associations.
Coordinated functioning of these three elements (PMD and $\mathrm{MD}$ in the condition of effectively organized activity brings in three-repeated resonance in 3-6-9 cycle, which makes the whole system active) determines positive PCS dynamics, provides general PCS function (that is cognitive activity) and places system onto the upper (or the highest) organizational level. We call it the level of self organization. Uniting elements into the system causes the appearance of new system properties, which are not the properties of its components but belong to the system only and exist until this system is an indivisible union.

Thus in the process of investigation using MCALS we can obtain wide specter of indices, which describe individual peculiarities of education progress, thinking productivity and elements structural organization specific of students' PCS.

Theoretic and methodological basis of MCALS. The first methodological principal is based on self organizational systems theory, namely it is based on cycle dynamic concept of PCS self organization. The coordinated, harmonic components correlation in subsystems (in the systems of the second, the third order) with constantly high structure-and-functional level brings out PCS onto the functional self organization level. The criterion of self organization is high mental functioning productivity.

The second methodological principal was formulated on the ground of kolmogorov complexity theory, and its idea is that the sound speech aspect is subordinated to ordinary statistic mechanisms, which can be calculated with the help of probability theory [4].

Letter-signs usage frequency in oral or written speech allows to form individual sign frequency vocabulary, in which investigated text letter-sign specter is represented. Individual frequency parameters of letter-sign specter are unique and represent personal psychic instruments peculiarities. The main point in abilities development understanding is thesis about the sign facilities role in psychological functions reconstruction and including them into the more complex structural unions. The ability development process is integrative formation, in the center of which there is a sign (word). Thus integration degree positively influences individual thinking productivity and is revealed quantitatively and structurally in frequency characteristics of SS sign markers. The system is used by a person during original text creation. SS are the main social and cultural factors in providing a person with thinking productivity.

In our investigation we used the hypothesis that students' SS signs and words individual frequency parameters are correlated with their thinking productivity $[3,5]$. The general method of investigation was the quantitative analysis of creative activity products and the method of objective expert estimation of students' knowledge obtained during the General State Examination (GSE). The methods served as measure and investigation of correlation between individual sign specter frequency markers and personal cognitive abilities functional 
development level markers in the time when students entered High schools.

The analysis and interpretation of the results obtained were statistically processed with the use of STATISTICA 6.0 for Windows. On the basis of SS signs frequency analysis of 11 grade students' texts personal letter frequency vocabularies were formed. Quantitative frequency location of signs obtained during the analysis of all texts was represented in two variants: in the order of sign frequency decrease and in the form of spectral letter frequency vocabulary. These spectral letter frequency vocabularies are the ground for the investigation of separate signs frequency parameters correlation with thinking productivity parameter. The presence of meaningful connections between individual thinking productivity parameters and SS signs frequency parameters show that thinking productivity is connected with frequency characteristics of consonants number. For example, "б" $(\mathrm{r}=0,39)$, " $\mathrm{r}$ " $(\mathrm{r}=0,30)$, “ $"(\mathrm{r}=0,33)$, “л" $(r=0,23)$, " $\mathrm{"}(r=0,36)(p<0,005)$ etc. Groups with low and high indices of thinking productivity differ in SS structure. In the groups with productivity thinking high indices the sum of strongly connected consonants and vowel is $40,96 \%$, that is in 1,8 times more than those of in the group with low indices of thinking productivity. The number of important correlations in pares consisting of consonants only is conversely in 1,2 times more in the group with low indices of thinking productivity. The present differences are the evidence of SS structures different variants in contrasting groups.

The third methodological principle. In written speech each alphabet sign is a finished picture or scheme. The abstract sign image integrity in human consciousness is conditioned by the three correlated elements (semantic concepts) combination. The first one is representation on different modality levels. The second is expression. The third is appeal [4]. According to the formulated principle we used psycho semantic approach described and proved by $\mathrm{K}$. Buhler and P.V. Yanishev [6,7]. This approach allows to unite the phenomena of color perception and representation, color influence on emotional and physiological state and mechanisms of color associations and color attribution. Considered as an image color has its subject matter revealed in subject meanings [6,7]. According to the perception phenomenon the image color scheme reflects the emotions state of a person. According to the color attribution phenomenon the subject matter of color scheme is emotional attitude to a letter-sign. In both causes the color image in equal to utterance.

\section{CONCLUSION}

The results obtained have important theoretical and practical value.

For cognitive psychology according to the individual thinking productivity managing aim the correlation of objectively measured individual thinking productivity with individual SS spectrally measured parameters was found.

Application for the social and practical sphere is expressed in the following:

1. MCALS, the technology of complex investigation with the use of letter-sing color association was invented.

2. The correlation of natural and social aspects was revealed. This correlation is carried out by the mechanism of color standards and letter-sings associations:

- Color perception is fully subdued to image production mechanisms (K. Buhler);

- Actual psychological person state by means of color preference analysis following M. Lusher tests;

- On the basis of mechanism of verbal and non-verbal aspects associative combination of neuron psychic brain activity the $\mathrm{Me}-\mathrm{Conception}$ characteristics are revealed. Meconception characteristics are Me-actual and Meunrealized;

- Individual psychology peculiarities are revealed in sensorperception adequacy measuring.

3. The problems of professional orientation optimization in the sphere on intellectual careers and on different levels can be solved easily.

\section{REFERENCES}

[1] L.V. Akhmetova. Structural self-organization of the determinants of the cognitive sphere of the personality of younger schoolchildren. The processes of self-organization in the Universal History: International Symposium (Belgorod, September 29 - October 2, 2006). - Belgorod: Publishing house BelGu. 2004, pp.158-160.

[2] L.V. Akhmetova, V.G. Morogin. Determinants of cognitive development of the personality of primary school pupils. Humanitarian research and their role in the development of pedagogical education: the International scientific-practical course. Conf. (Tomsk, November 4-6, 2002). - Tomsk: Publishing house of TSPU, 2004, pp.195-200.

[3] L.V. Akhmetova. Productivity of thinking in the aspect of individual features of the structural organization of the cognitive sphere of the personality of students // Bulletin of the Tomsk State Pedagogical Institute. University. Issue. 2 (53). Ser. Psychology. Tomsk: Publishing house of TSPU, 2006, pp. 34-39.

[4] A.N. Kolmogorov. Three approaches to the definition of the concept of "amount of information" / / Problems of information transfer. 1965.V.1.№ 1.pp.25 - 38.

[5] L.V. Akhmetova, V.A. Ostrovsky. The frequency parameters of the personality SSS elements as correlates of the productivity of thinking. Language and Culture: The 18th International Scientific Conference. (Tomsk, 18-20 April 2005). - Tomsk: Izd-vo TSU, 2006, pp. 20-23.

[6] K. Bühler. Theory of Language. - Moscow: Progress, 1993, 504 p.

[7] V.P. Yanshin Psychosemantic. analysis of color categorization in the structure of the subject's consciousness. Author's abstract of doc. Crazy. sciences. - Moscow, $2001-2.0$ pp. Publishing house of the Samara GPU. 